\title{
DISCUSSION
}

\section{Bearing strength surfaces implied in conventional bearing capacity calculations}

\author{
MICHAEL JOHN PENDER* and CHRISTOS VRETTOS $†$
}

\section{Contribution by Christos Vrettos}

The author derived analytical expressions that transform the classical three-term bearing capacity equation into an equivalent three-dimensional bearing strength surface (BSS) (Pender, 2017). Besides enhanced physical insight, this representation provides the means for an alternative definition of the available safety margin as the distance of the current loading state to the BSS. The discusser very recently followed the same concept and obtained these surfaces by a numerical solution of the respective bearing capacity equations (Vrettos \& Seibel, 2017). By inspecting the graphs, a discrepancy appeared for the undrained case that was the motivation to look more thoroughly into the derivation of equation (4).

Starting from the seventh equation in Appendix 2 of the paper

$$
\mathrm{Vn}=\frac{1}{2}\left(1-\frac{2|\mathrm{Mn}|}{\mathrm{Vn}}\right)\left[1+\sqrt{1-\frac{|\mathrm{Hn}| N_{\mathrm{c}}}{(1-2|\mathrm{Mn}| / \mathrm{Vn})}}\right]
$$

It can be seen that the specific inclined load factor $\lambda_{\text {ci }}$ defined by equation (31) contains a square root, thus implying that the radicand must always be either positive or zero. In other words, it is a priori required that the resistance along the foundation-soil interface is sufficient to preclude sliding failure. For the strip foundation considered this implies

$$
H \leq B^{\prime} s_{\mathrm{u}}
$$

Hence, the maximum horizontal shear force is

$$
\mathrm{Hn}_{\max }=\left(1-2 \frac{|\mathrm{Mn}|}{\mathrm{Vn}}\right) \frac{1}{N_{\mathrm{c}}}
$$

First, consider the special case $|\mathrm{Mn}|=0$. For given $0<\mathrm{Vn}<1$ values $\mathrm{Hn}$ are sought that satisfy exactly the bearing capacity equation. It is evident that no solution is possible for $\mathrm{Vn}<0 \cdot 5$ since the radicand cannot be negative. Hence, the maximum allowable horizontal shear force in this range is $\mathrm{Hn}_{\max }=1 / N_{\mathrm{c}}=1 /(2+\pi)$.

For $|\mathrm{Mn}|>0$ the radicand in equation (38) becomes zero, and accordingly $\mathrm{Hn}$ is bounded by $\mathrm{Hn}_{\max }$, only when $\mathrm{Vn}$ satisfies the following equation

$$
2 \mathrm{Vn}^{2}-\mathrm{Vn}+2|\mathrm{Mn}|=0
$$

with roots

$$
\mathrm{Vn}_{1,2}=\frac{1 \pm \sqrt{1-16|\mathrm{Mn}|}}{4} \mathrm{Vn}_{1} \geq \mathrm{Vn}_{2}
$$

\footnotetext{
* Department of Civil and Environmental Engineering, University of Auckland, Auckland, New Zealand.

$\dagger$ Technical University of Kaiserslautern, Kaiserslautern, Germany.
}

For $\mathrm{Vn}_{2}<\mathrm{Vn}<\mathrm{Vn}_{1}$ the requirement of sufficient sliding resistance $\mathrm{Hn} \leq \mathrm{Hn}_{\max }$ dominates. For example, for $|\mathrm{Mn}|=0$ this affects the range $0<\mathrm{Vn}<0 \cdot 5$, for $|\mathrm{Mn}|=0.06$ the range $0 \cdot 2<\mathrm{Vn}<0 \cdot 3$, whereas for $|\mathrm{Mn}|>1 / 16$ no limitation prevails. On the other hand, for vertical load smaller than the smallest root of equation (42) bearing failure becomes relevant due to the large load eccentricity.

In the $\mathrm{Vn}-|\mathrm{Mn}|$ cross-section of the BSS, the requirement $\mathrm{Hn} \leq \mathrm{Hn}_{\max }$ leads to a bound for the maximum allowable bending moment, $|\mathrm{Mn}| \leq|\mathrm{Mn}|_{\max }$. From equation (40), the following is obtained

$$
|\mathrm{Mn}|_{\max }=\frac{\mathrm{Vn}}{2}\left(1-\operatorname{Hn} N_{\mathrm{c}}\right)
$$

For $\mathrm{Hn}=\mathrm{Hn}_{\max }$ equation (31) yields $\lambda_{\mathrm{ci}}=0 \cdot 5$. Inserting this value into the bearing capacity equation it follows that $|\mathrm{Mn}|_{\max }$ according to equation (43) constitutes the upper bound of the bending moment as long as the vertical load is smaller than a threshold value given in equation (44)

$$
\mathrm{Vn} \leq \frac{\operatorname{Hn} N_{\mathrm{c}}}{2}:|\mathrm{Mn}| \leq \frac{\mathrm{Vn}}{2}\left(1-\operatorname{Hn} N_{\mathrm{c}}\right)
$$

In the limiting case $\mathrm{Hn}=0$ no bound exists, and the maximum sustainable moment is derived from equation (38): $|\mathrm{Mn}|_{\max }=1 / 8$ at $\mathrm{Vn}=0 \cdot 5$.

In summary, the ultimate limit state of sliding $\mathrm{Hn}=\mathrm{Hn}_{\max }$, with $\mathrm{Hn}_{\text {max }}$ from equation (40), dominates over bearing failure when one of the following two conditions prevails: either (a) $\mathrm{Vn}_{2}<\mathrm{Vn}<\mathrm{Vn}_{1}$, or (b) inequality (44) holds. This is the direct consequence of the particular expression used for the load inclination factor. Hence, equation (4) in the paper is valid only when neither of the two conditions $(a)$ and $(b)$ is met. In deriving equation (4) the author eliminated the square root by squaring equation (38) but ignored the sign of the radicand, thus introducing solutions that are not feasible. Fig. 12 displays contour lines in the $H-V$ and $M-V$ sections of the normalised BSS.

As for the drained case, attention is required when evaluating equation (7) for load combinations lying underneath the respective maximum BSS, as depicted in Fig. 13. The starting and end points of the curves on the Vn-axis are approaching each other as the loading intensity increases. The cubical term in equation (7) introduces, besides the dominant BSS at higher values of $\mathrm{Vn}$, an additional BSS at lower values of $\mathrm{Vn}$ that must be rejected, being physically not meaningful. At the limiting cases given by equations (8) and (9), the numerical values of the maximum sustainable loading (crest of BSS) can be derived after some algebra: $\mathrm{Hn}_{\max }=27 / 256$ for $|\mathrm{Mn}|=0 ;|\mathrm{Mn}|_{\max }=54 / 729$ for $\mathrm{Hn}=0$.

The restrictions to equation (4) for the undrained case highlighted above have implications in practice: as in foundation designs $\mathrm{Vn}<0.5$, verification against sliding failure will be the decisive check in many cases. 

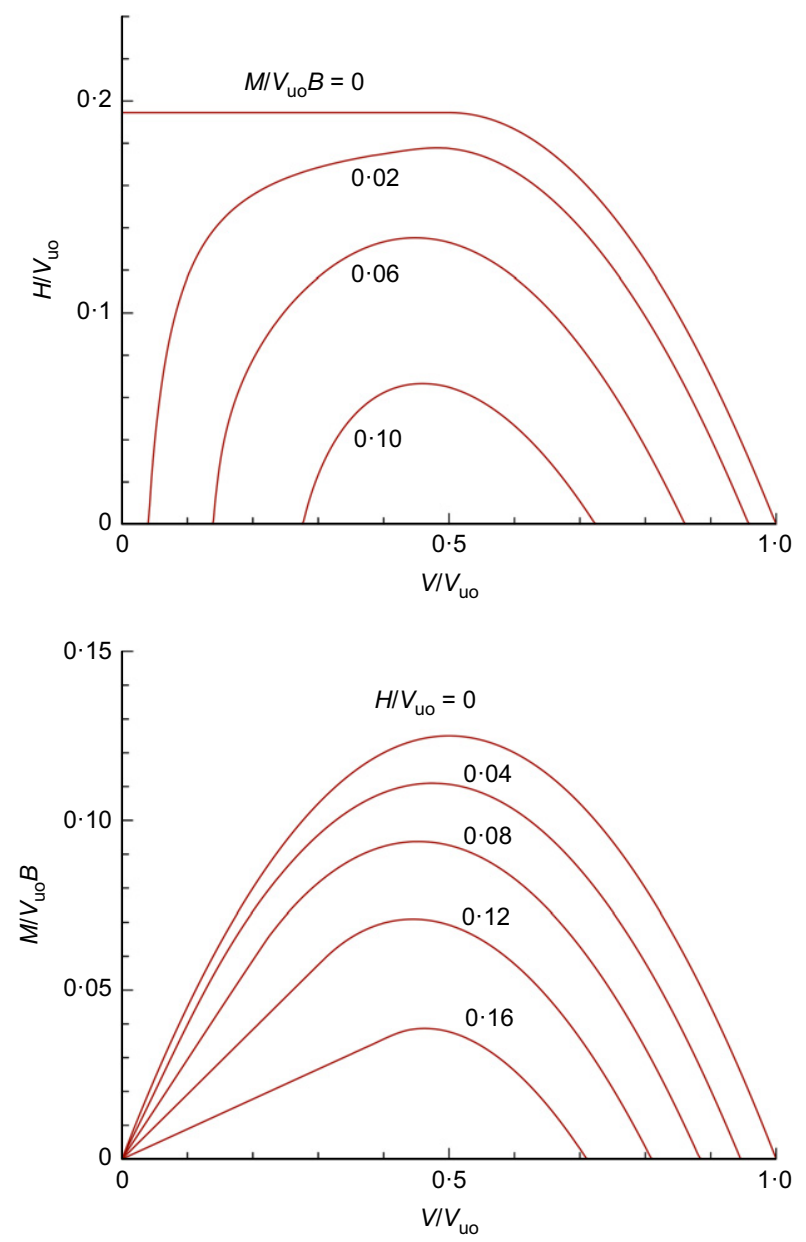

Fig. 12. Contour lines in the $H-V$ and $M-V$ sections of the BSS for the undrained case

Finally, it should be pointed out that the broad adoption of the BSS concept in routine design will require the incorporation of both cohesion and friction for the soil, and of non-vanishing lateral surcharge pressure $q$. Analytical expressions for this general case are cumbersome to derive, and a numerical solution may be used instead.

\section{Author's reply}

The author offers his thanks to Professor Vrettos for his interest in the paper, which, as indicated in the title, refers to shallow foundation bearing strength. Professor Vrettos is quite correct to point out that the sliding resistance of the foundation needs consideration as well as the bearing strength. The discussion focuses on the case of a surface strip foundation on saturated clay - that is, the undrained bearing strength surface.

Towards the end of his contribution, the discusser suggests that the author made an error in squaring the BSS equation developed to remove the square root associated with $\lambda_{\mathrm{ci}}$

$$
\lambda_{\mathrm{ci}}=0 \cdot 5\left(1+\sqrt{1-H / A s_{\mathrm{u}}}\right)
$$

This decision was simply a matter of personal taste; to my eye the equation without the square root 'looks better'. Such an operation would be erroneous if both the positive and negative square roots were required in bearing strength calculations. As only the positive root is used in such calculations, there is no error in the procedure that the
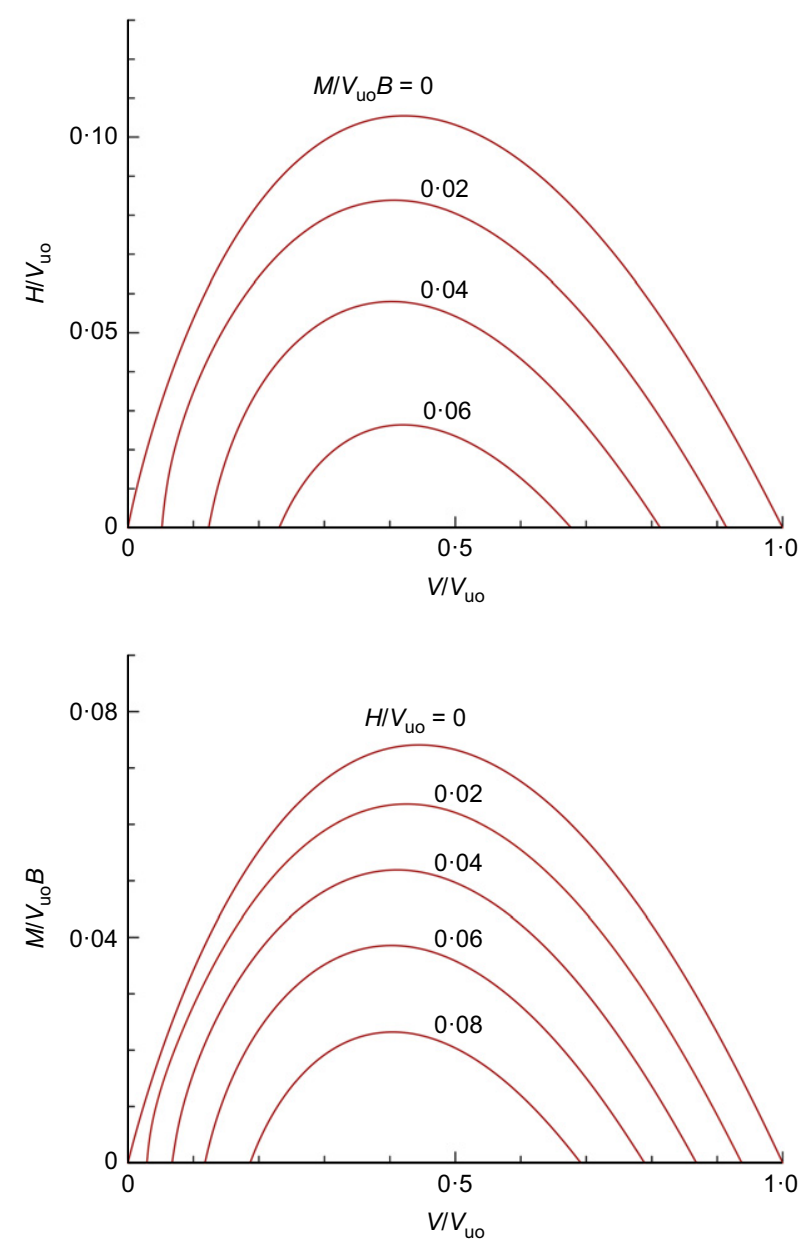

Fig. 13. Contour lines in the $H-V$ and $M-V$ sections of the BSS for the drained case

author followed. In fact, the plotted bearing surface is the same if no squaring is done.

When a foundation is subject to moment the contact area between the underside of the foundation and the soil below is reduced, that is $B^{\prime}<B$. The shear force applied to the foundation must satisfy the following inequality if sliding failure is not to occur (assuming that the shear resistance at the interface is the full value of the undrained shear strength of the clay)

$$
H<B^{\prime} s_{\mathrm{u}}
$$

Figure 14(a) is a cross-section of the undrained bearing strength surface for a normalised vertical load of $0 \cdot 20$ (i.e. the applied vertical load on the strip foundation is $20 \%$ of vertical load only bearing strength). Every point on this cross-section gives a combination of moment and shear which will induce bearing failure of the foundation at the fixed vertical load.

Now it is simple enough to calculate the available shear capacity at each point around the cross-section. Herein this is done for the specific case of a surface strip foundation $3 \mathrm{~m}$ wide on saturated clay with an undrained shear strength of $50 \mathrm{kPa}$. The moment gives a reduced foundation width from which the sliding resistance can be calculated using equation (39) above, substituting equality for the less than sign. Fig. 14(b) gives the results of this calculation, from which it is clear that, except for one point, the sliding resistance of the foundation, $B^{\prime} S_{\mathrm{u}}$, plots on a straight line which lies above the BSS. The one exception is the point marked with a filled circle, where the shear capacity line is tangential for the BSS, 


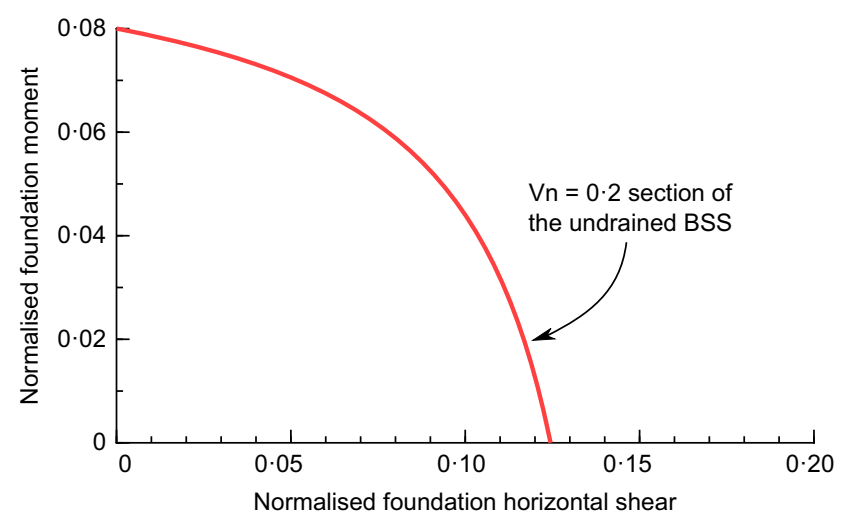

(a)

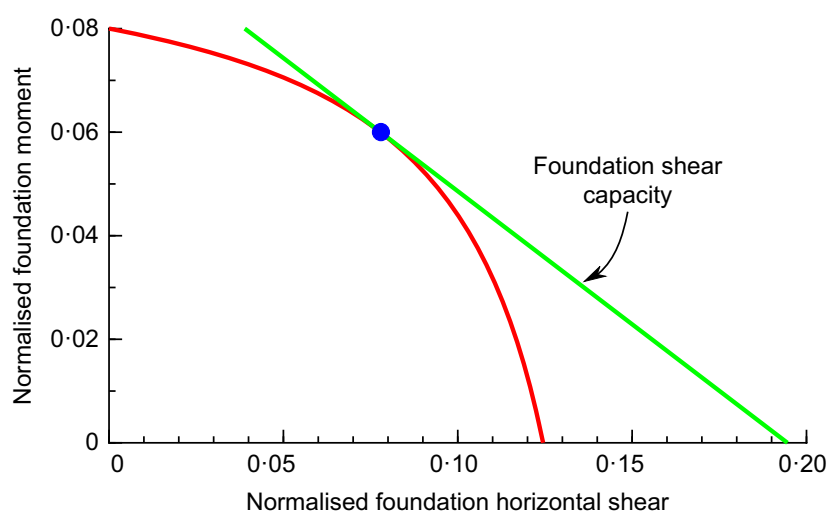

(b)

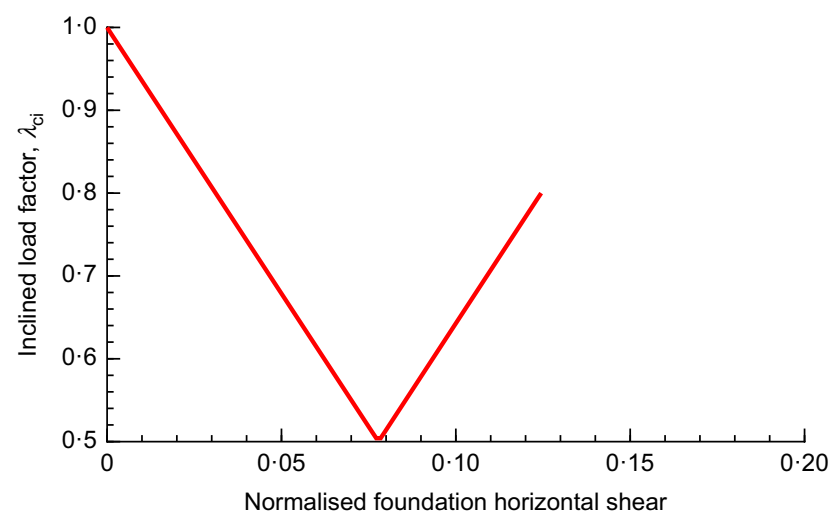

(c)

Fig. 14. Vn=0.2 cross-section of the undrained BSS: (a) the Mn-Hn cross-section; (b) the Mn-Hn cross-section with the foundation shear capacity plotted and the point in common with both relations indicated; (c) the variation of $\lambda_{\text {ci }}$ around the cross-section
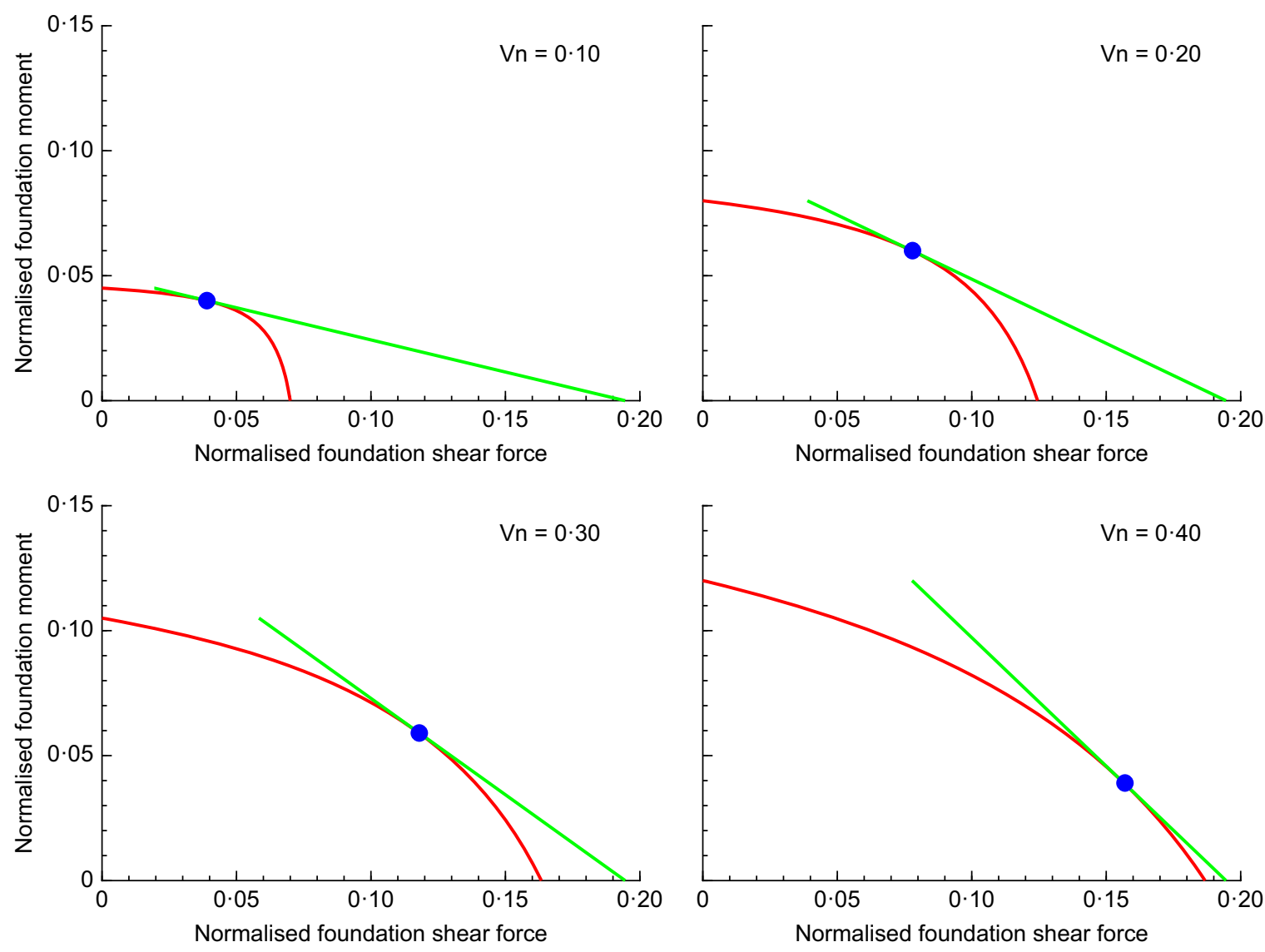

Fig. 15. Constant Vn cross-sections of the undrained BSS for $\mathrm{Vn}=0 \cdot 1,0 \cdot 2,0 \cdot 3$ and $0 \cdot 4$ (curve - BSS, tangent - normalised shear capacity) 
so coupled bearing and sliding failure would occur at that point. If the shear failure line is extended to $M n=0$, the line intersects the horizontal axis at $1 / N_{\mathrm{c}}(0 \cdot 195)$.

Next, it is of interest to plot the behaviour of $\lambda_{\mathrm{ci}}$ around the above cross-section, Fig. 14(c). This reveals that $\lambda_{\mathrm{ci}}$ does not have a uniform decrease as the shear force increases, but reaches a minimum value at some point during the loading after which it increases again. The value of 0.5 corresponds to the square root bracket in $\lambda_{\mathrm{ci}}$ reaching zero.

Figure 15 has cross-sections of the undrained BSS for $\mathrm{Vn}=0 \cdot 4,0 \cdot 3,0 \cdot 2$ and $0 \cdot 1$. In each case, the line giving the sliding resistance is also plotted. It is apparent that the behaviour plotted in Fig. 14(b) is seen for all values of Vn less than $0 \cdot 5$.
Thus, it appears that the discussion contributor's approach to foundation sliding resistance is unnecessarily restrictive.

The author offers his thanks again to Professor Vrettos for bringing this question to his attention.

\section{REFERENCES}

Pender, M. J. (2017). Bearing strength surfaces implied in conventional bearing capacity calculations. Géotechnique 67, No. 4, 313-324, https://doi.org/10.1680/jgeot.16.P.024.

Vrettos, C. \& Seibel, E. (2017). Bearing capacity of strip footings in regions of medium seismicity: reappraisal of the pseudostatic approach in code-based design in light of recent computational results. Proceedings of the $3 r d$ international conference on performance-based design in earthquake geotechnical engineering, Vancouver, Canada, paper 204. 